\title{
Selfish Mules: Social Profit Maximization in Sparse Sensornets using Rationally-Selfish Human Relays
}

\author{
Shusen Yang, Usman Adeel, Julie A. McCann \\ Department of Computing, Imperial College, London, United Kingdom \\ \{s.yang09, u.adeel09, j.mccan\}@imperial.ac.uk
}

\begin{abstract}
Future smart cities will require sensing on a scale hitherto unseen. Fixed infrastructures have limitations regarding sensor maintenance, placement and connectivity. Employing the ubiquity of mobile phones is one approach to overcoming some of these problems. Here, mobility and social patterns of phone owners can be exploited to optimize data forwarding efficiency. The question remains, how can we stimulate phone owners to serve as data relays? In this paper, we combine network science principles and Lyapunov optimization techniques, to maximize global social profit across this hybrid sensor and mobile phone network. Sensor data packets are produced and traded (transmitted) over a virtual economic network using a lightweight socialeconomic-aware backpressure algorithm, combining rate control, routing, and resource pricing. Phone owners can get benefits through relaying sensor data. Our algorithm is fully distributed and makes no probabilistic/stochastic assumptions regarding mobility, topology, and channel conditions, nor does it require prediction. The global social profit achieved by our algorithm can perform close to (or better than) an ideal algorithm with perfect prediction- proven by rigorous theoretical analysis. Simulation results further demonstrate that the proposed algorithm outperforms pure backpressure and social-aware schemes; highlighting the advantage of building systems combining communication with other types of networks.
\end{abstract}

Index Terms-Network Optimization, Economic Networks, Social Networks, Delay Tolerant Networks, Incentive, Human mobility, Smart Cities.

\section{INTRODUCTION}

Complex networks, such as social, economic, and biological, are having significant impacts on the field of computer communications. Such networks exhibit complexity in terms of heavy-tail degree distributions [1], small-world phenomenon [2], community structures [3], [4], etc. The close coupling between modern mobile and sensing networks and the physical world in which they reside, has lead to the nontrivial topological features of such complex networks being exploited in the design of communication schemes in computer networks [5]-[7].

In this paper, we apply network science principles to build a resilient architecture consisting of a hybrid of mobile wireless devices and wireless sensor networks (WSN). Here static sensors are deployed to instrument a space and report sensed readings. However, we deviate from the traditional WSN architecture by not using end-to-end paths for sensor data transmissions, but instead utilize human relays via their mobile devices. The motivating application of such an architecture is a Sustainable Smart City [8]-[10]. For brevity we call our architecture WSN-HR (Wireless sensor networks with Human Relays).

\section{A. Motivation}

Urban sensing [8]-[10] is becoming ubiquitous; from light and temperature sensors in a smart building, water leak detection in the sewer, to noise and air-quality monitoring in the streets. Some are able to connect to the Internet directly or through a connected multi-hop WSN, but this may be prohibitive in some instances due to high costs or low communication coverage. For instance, $3 \mathrm{G}$ cellular data communications can be expensive for large volumes of delaytolerant sensor data (e.g. several megabytes [8]), while free $\mathrm{WiFi}$ connections are not always available.

An attractive alternative is to use the prevalence of mobile phones and the opportunistic communication paradigm [9]. Here, the mobile phones act as data relays to bridge the connectivity gap between the sensors and base-stations (sinks) by leveraging low-cost short-range communications (e.g. Zigbee, WiFi direct, and Bluetooth) and human mobility [8], [9]. Such low cost opportunistic communication paradigms are especially useful for delay-tolerant sensing applications (e.g. urban traffic monitoring). For a WSN-HR to be a costeffective communication solution for future smart sustainable cities, the following two key issues must be addressed:

- Since the mobility patterns of human relays are governed by their underlying social networks [5], [11], how can we exploit social network features, such as centrality and community, for efficient sensor data forwarding?

- Since using the mobile phone as a relay costs, in terms of local resources (e.g. memory and energy) and telecommunications, how can we incentivise individuals to participate?

\section{B. Our Approach}

To address the above, we develop a novel scheme, a Backpressure approach with Social/Economic Awareness (termed OBSEA in this paper), for joint rate control, flow routing, and resource pricing, that uniquely combines network science principles and Lyapunov stochastic optimization theory [12]-[14]. Specifically, the contributions of this paper are summarized as follows

1. By exploiting mobility patterns and the underlying social graph of human relays, we propose a novel data forwarding 
metric, Sink-Aware (SA) centrality, to measure the potential sensor data forwarding ability of mobile relays.

2. To incentivise people to serve as a data relay using their phone, we establish a virtual economic network for sensor data production and trading. Here, the static WSN consisting of fixed sensors and sinks, makes a profit by producing sensor data to maximize the network utility, and each mobile relay acquires profit by dynamically adjusting the selling price of its maintained sensor data and trades (transmits and receives) data with others opportunistically at each moment of contact.

3. We formalize a finite-horizon optimization problem to maximize the global social profits of the all nodes in the WSNHR. Our formalization does not make any probabilistic/stochastic assumptions (e.g. specific probability distributions or ergodicity) for the network conditions (e.g. mobility, topology, and wireless channel), and thus is suitable for the arbitrary dynamic evolution process of WSN-HR. The lightweight OBSEA solves this problem using only current and local information. This means that OBSEA is fully distributed and does not require any prediction capacities, therefore maximizing the practical application of the work.

4. Through rigorous analysis, we show that OBSEA can avoid buffer overflow for every node, and the social profit of the non-predictive OBSEA algorithm can be arbitrarily close to an ideal algorithm with prefect future network knowledge. We evaluate the performance of OBSEA by using the Castalia [15] simulator and a realistic mobility model [11], which exhibits features observed from real social networks and human mobility traces. Simulation results demonstrate that OBSEA is adaptive to different network settings and outperforms pure backpressure routing and pure social-aware forwarding scheme; in terms of global social profit, data buffer efficiencies, and end-to-end delays. In addition, the results also show that a "win-win situation" (non-negative benefits) can be achieved by both the static WSN and all mobile phone owners.

\section{Related Work}

Data Muling for WSNs. Many data muling schemes have been proposed to improve energy efficiency and coverage in sparse WSNs. Specifically, [8], [16]-[19] recognize the potential of human mobility in data mules. As far as we are aware, however, none of them consider exploiting the underlying social networks of human relays or utilizing opportunistic multi-hop human contacts.

Intermittently Connected Networks and Social Awareness. Social network metrics, centrality and community structure, have been used for many opportunistic routing schemes [5]-[7], [20], [21] in Delay Tolerant Networks (DTNs) [22]. However, all of them focus on individual packet routing rather than routing and control for streams of packets as for our OBSEA. Backpressure-type algorithms exist [23], [24] for intermittently connected networks. They are based on assumptions of predetermined gateways and ergodic network conditions (e.g. The assumption of Markov mobility process). However, our work uses a much more general and realistic network model in which any sensor could serve as a gateway at any opportunistic contact, and no probabilistic/stochastic as- sumptions are made for arbitrary network conditions. Further, none of them consider social or economic awareness.

Network Optimization. Stochastic Cross-layer network optimization and control is an active networking research area [13], [14]. Most backpressure scheduling/routing schemes [25]-[27] are developed for multi-hop wireless networks to achieve infinite-horizon stability, and to maximize long-term network utilities or minimize costs. However, these schemes are limited to ergodic network models, which may not hold true in our highly dynamic WSN-HR. The recent universal scheduling framework developed by Neely [28] optimizes finite-horizon general network utility with arbitrary dynamic network processes, which has been used in $\mathrm{P} 2 \mathrm{P}$ networks [29] and smart electricity markets [30]. OBSEA is the first to combine the universal scheduling framework [28] and network science principles and then applying it urban WSNs using data mules.

Pricing, Incentive, and Selfishness. In [29], [31], [32], Lagrange multipliers or queue backlogs are used as prices to solve static convex network problems or dynamic stochastic problems. However, OBSEA uses both queue backlog and social-aware metrics for pricing. [33]-[35] study gametheoretic incentive and pricing approaches. Incentive-aware routing [36], [37] are proposed for data forwarding in DTNs. DTN routing schemes [38], [39] consider the concept of social selfishness, which means that an individual is willing to provide better service to those with strong social ties than those with weaker social ties. However, rational selfishness considered in our work means that a phone owner is willing to relay sensor data as long as he or she can benefit, which is conceptually different form social selfishness. In addition, none of above schemes focus on data muling for WSNs.

\section{Paper Organization}

The next section presents the network model. Section III describes the proposed OBSEA algorithm. Theoretical analysis and simulation are presented in Sections IV and V respectively. Finally, we conclude the paper in Section VI.

\section{NETWORK MODEL}

In an intermittently connected WSN-HR, every sensor node collects environmental data (e.g. temperature and humidity) and sends the sensor data to any of the $\operatorname{sink}(s)^{1}$ through other static sensor nodes and mobile relays if necessary, in an opportunistic multi-hop manner.

\section{A. Topology and Communication model}

Let the sets of sensor nodes, human relays, and sinks be $\mathcal{S}, \mathcal{R}$, and $\mathcal{D}$ respectively. Denote $\mathcal{N}=\mathcal{S} \cup \mathcal{R} \cup \mathcal{D}$ as the set of all nodes in the WSN-HRs. The network operates in discrete time with a unit time slot $t \in\{1,2, \ldots\}$. Let $c_{x, y}(t) \geq 0$ be the current capacity of wireless link from node $x \in \mathcal{N}$ to node $y \in \mathcal{N}$ at time $t$, i.e. the maximum (integer) number of sensor data packets that can be successfully transmitted from $x$ to

\footnotetext{
${ }^{1}$ For brevity, this paper only considers the anycast traffic model. However, it is straightforward to extend OBSEA to the multi-commodity traffic model.
} 


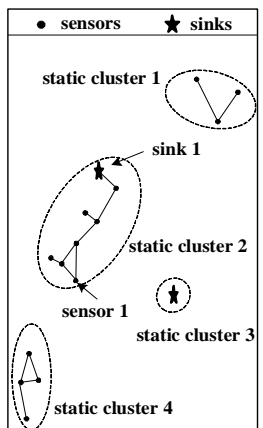

(a)

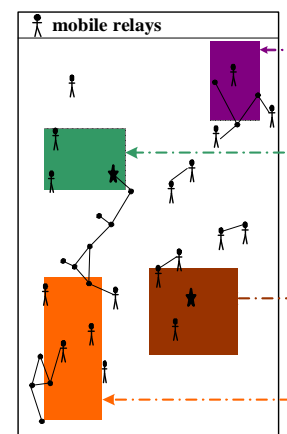

(b)

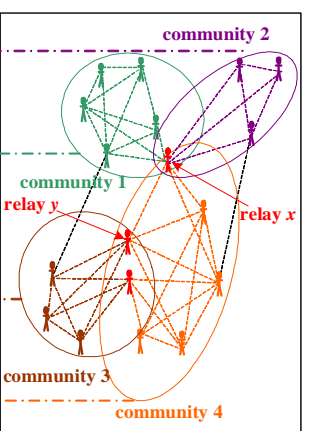

(c)
Fig. 1. Conceptual illustration of a WSN-HR. (a) Four static clusters of a static disconnected WSN $G^{w s n}\left(\mathcal{S} \cup \mathcal{D}, \mathcal{L}^{w s n}\right)$. (b) An example of instantaneous graph $G(\mathcal{N}, \mathcal{L}, \boldsymbol{c}(t))$ at a slot, and community-location mapping. Solid lines between nodes represent the wireless links with nonzero capacities, and the wireless links with zero capacity are not plotted. The colorful rectangles indicate the geographic areas associated with the communities. (c) The underlying social network consisting of mobile relays, and an example of 4-clique overlapping community structure [3] over the social network.

$y$ during slot $t . c_{x, y}(t)$ is assumed to be constant within the duration of a slot, but can vary from slot to slot and across different wireless links. Specifically, if $c_{x, y}(t)>0$, we say nodes $x$ and $y$ are in contact at slot $t$; otherwise, they are not in contact at slot $t$.

We model the whole WSN-HR as a directed, complete, and time-varying weighted graph $G(\mathcal{N}, \mathcal{L}, \boldsymbol{c}(t))$, where $\mathcal{L}=\{(x, y) \mid x, y \in \mathcal{N}\}$ represents the set of all possible wireless links between each pair of nodes in $\mathcal{N}$, and the $|\mathcal{L}|$-dimensional vector $\boldsymbol{c}(t)$ represents the vector of channel capacities over all wireless links at slot $t$. Figure 1(b) illustrates an example of instantaneous $G(\mathcal{N}, \mathcal{L}, \boldsymbol{c}(t))$ at a slot.

We do not make any probabilistic/stochastic assumptions on $\boldsymbol{c}(t)$, such as specific probability distribution, i.i.d., or even ergodicity. This is because the stochastic process $\boldsymbol{c}(t)$ could be affected by many random time-varying events such as unexpected external interference, channel fading, and human mobility, governed by various complex physical rules. It is easy to see that the definition of $G(\mathcal{N}, \mathcal{L}, \boldsymbol{c}(t))$ is very general and can characterize arbitrary stochastic channel states and topology processes (e.g. mobility) of the $|\mathcal{N}|$-node WSN-HR.

To focus on routing and rate control (also due to the sparsity of the network), we do not consider scheduling for wireless interference in the theoretical analysis. However it is straightforward to integrate distributed scheduling into our OBSEA for practical CSMA radios by using existing approaches such as [40], which will be studied in our simulation.

For notation brevity, we also define $G^{w s n}\left(\mathcal{S} \cup \mathcal{D}, \mathcal{L}^{w s n}\right)$ to represent the static WSN, where $\mathcal{L}^{w s n}=\left\{(x, y) \mid c_{x, y}(t)>\right.$ $0 \forall t \geq 1, \forall x, y \in \mathcal{S} \cup D\}$ is the set of all wireless links with non-zero capacities between static nodes.

Definition 1. [Static Cluster.] A static connected cluster $\mathcal{A} \subseteq$ $\mathcal{S} \cup \mathcal{D}$ is defined as the set of all static nodes in a connected component $^{2}$ of the static WSN $G^{w s n}\left(\mathcal{S} \cup \mathcal{D}, \mathcal{L}^{w s n}\right)$.

\footnotetext{
${ }^{2}$ In graph theory, a connected component of a graph $G$ is a subgraph in which any two vertices are connected to each other by paths, and which is connected to no additional vertices in $G$.
}

For instance, there are four static clusters in the WSN shown in Figure 1 (a).

\section{B. Queueing Dynamics}

Each node $x \in \mathcal{N}$ maintains a queue (i.e. data buffer) for the sensor data, which stores the data packets generated by itself (if $x$ is a sensor node), and by other sensor nodes. Let $Q_{x}(t) \geq 0$ be the queue backlog (or queue length) of $x \in \mathcal{N}$ at slot $t \geq 1$. Let $\mathcal{N}_{x}(t) \subset \mathcal{N}$ be the set of nodes that are in contact with node $x$ at slot $t$, i.e. the set of $x$ ' all instantaneous neighbors. For each node $x \in \mathcal{N}$, its queue backlog updates from slot $t$ to $t+1$ as follows:

$Q_{x}(t+1)=$

$$
\begin{cases}0 & x \in \mathcal{D} \\ \left|Q_{x}(t)+\sum_{y \in \mathcal{N}_{x}(t)}\left(f_{y, x}(t)-f_{x, y}(t)\right)\right|_{+} & x \in \mathcal{R} \\ \left|Q_{x}(t)+r_{x}(t)+\sum_{y \in \mathcal{N}_{x}(t)}\left(f_{y, x}(t)-f_{x, y}(t)\right)\right|_{+} & x \in \mathcal{S}\end{cases}
$$

where $r_{x}(t) \geq 0$ is the sensing rate at which a sensor node $x \in \mathcal{S}$ collects environmental data at slot $t, 0 \leq f_{x, y}(t) \leq$ $c_{x, y}(t)$ represents the actual amount of data transmitted from node $x$ to node $y$ at slot $t$, and for any real number $a$, the operator $|a|_{+}=a$ if $a>0 ;|a|_{+}=0$ otherwise.

\section{Mobility Pattern and Social Network of Human Relays}

The following human mobility and social network properties are explored by our OBSEA algorithm.

Pairwise Inter-Contact Time. Let $I C T_{x, y}$ be the intercontact time (i.e. the time elapsed between two successive contacts) between a pair of relays $x, y \in \mathcal{R}$. The distribution of pair wise inter-contact times between mobile relays has a great impact on data forwarding [41]. We do not assume any special distribution for $I C T_{x, y}, x, y \in \mathcal{R}$ (e.g. power-law [41], exponential [21], or power-law head and exponential tail [42]). In addition, we generalize the concept of inter-contact time from each pair of pure mobile relays in $\mathcal{R}$ to that of all nodes in $\mathcal{N}$. Specifically, if two static nodes $x, y \in \mathcal{S} \cup \mathcal{D}$ are always in contact (i.e. $c_{x, y}(t)>0$ for all $t \geq 1$ ), $I C T_{x, y}=0$; otherwise (i.e. $c_{x, y}(t)=0$ for all $\left.t \geq 1\right)$ ), $I C T_{x, y}=\infty$.

Overlapping Communities and Centrality. As shown in Figure 1 (c), we assume there is an underlying social network consisting of all mobile relays in $\mathcal{R}$. We model the social network as an undirected graph $G^{\text {social }}\left(\mathcal{R}, \mathcal{L}^{\text {social }}\right)$, where $\mathcal{L}^{\text {social }}$ represents the set of social ties between mobile relays, which can be defined by inter-contact time or contact probability between pairs of nodes. In this paper, a social tie between two mobile relays $x$ and $y$ in $\mathcal{R}$ is considered to exist, if the estimated average value of $I C T_{x, y}, \overline{I C T}_{x, y}$, is smaller than a predetermined threshold $\mathrm{ICT}^{\mathrm{max} 3}$. Each mobile relay $x \in \mathcal{R}$ maintains and updates its social neighbor table $\mathcal{N}_{x}^{\text {social }} \subseteq \mathcal{R}$, which is the set of mobile relays that share social ties with $x$. Using social neighbor table of each relay, the social network $G^{\text {social }}\left(\mathcal{R}, \mathcal{L}^{\text {social }}\right)$ can be established in a distributed way at runtime.

\footnotetext{
${ }^{3}$ In practice, $\overline{I C T}_{x, y}$ can be obtained at run time by using the EWMA
} approach or an arithmetic average of historical samples. 
It has been observed that a social network always exhibits overlapping community structures [3], [11], [43] and heterogeneous centrality [1], [6], [20]. In the WSN-HR context, overlapping community structure means that mobile relays in the same community (a set of mobile relays) meet each other much more frequently than that in different communities, and a mobile relay may belong to multiple communities, which can be detected at runtime by using the distributed approach in [43]. Heterogeneous centrality indicates that few mobile relays (e.g. postmen) meet a large number of other relays, but many mobile relays only meet a small number of others. We explore these two useful social network features in our OBSEA algorithm.

Mathematically, we use a tuple $\left(\mathbb{C}_{x}, \boldsymbol{h}_{x}\right)$ to represent the social profile of a mobile relay $x \in \mathcal{R}$, where $\mathbb{C}_{x}$ is the set of community(ies) that node $x$ belongs to, and $\boldsymbol{h}_{x}$ is a $\left|\mathbb{C}_{x}\right|-$ dimensional vector, where each entry $h_{x}^{i}, 1 \leq i \leq\left|\mathbb{C}_{x}\right|$ represents the local centrality [6], [11] of $x$ in its $i$ th community $\mathcal{C}_{x}^{i} \in \mathbb{C}_{x}$. In this paper, specifically, the $h_{x}^{i}$ is measured as the number of social ties between $x$ and other mobile relays in $\mathcal{C}_{x}^{i} \in \mathbb{C}_{x}$, i.e. $h_{x}^{i}=\left|\mathcal{C}_{x}^{i} \cap \mathcal{N}_{x}^{\text {social }}\right|$. For instance, in Figure 1 (c), the local centralities of relays $x$ and $y$ in community 4 are 3 and 4 respectively.

Spatial Regularity of Human Mobility. Recent observations [44], [45] of real human traces demonstrate that people in a given community normally move within some certain geographic areas much more frequently than other locations (e.g. students in the same department normally visit their department building with a much higher probability than other places). An example of such community-location mappings are illustrated in Figure 1 (b) and (c). Based on this property, we establish social awareness for each static node $x \in \mathcal{S} \cup D$, by using a variable $\mathbb{C}_{x}^{\text {static }}$, which represents the set of community(ies) associated with the geographic area where $x$ is located in. Take Figure 1 for instance, since sink 1 and sensor 1 are located within the area associated with communities 1 and 4 respectively, $\mathbb{C}_{\text {sink1 }}^{\text {static }}=\{$ community 1$\}$ and $\mathbb{C}_{\text {sensor } 1}^{\text {static }}=\{$ community 4$\}$.

\section{A Social Forwarding Metric: Sink-Aware Centrality}

Based on social networking features, we define a novel metric, Sink-Aware (SA) centrality, to measure the potential ability of a mobile relay for delivering sensed data to the sink. A distributed lightweight algorithm to establish SA centrality at runtime will be introduced in Subsection III A.

Let $\mathbb{A}^{\text {sink }}$ be the set of static clusters, each of which contains at least one sink:

$$
\mathbb{A}^{\operatorname{sink}}=\bigcup_{\mathcal{A} \in \mathbb{A},}\{\mathcal{A} \cap \mathcal{D} \neq \emptyset
$$

where $\mathbb{A}$ is the set of all static clusters. In Figure 1, for instance, $\mathbb{A}=\{$ static clusters $1-4\}$ and $\mathbb{A}^{\operatorname{sink}}=\{$ static clusters 2 and 3$\}$. We then define the global sink-aware community set $\mathbb{C}^{\text {sink }}$ as

$$
\mathbb{C}^{\text {sink }}=\bigcup_{x \in \mathcal{A},} \mathbb{C}_{x \in \mathbb{A}^{\text {sink }}} \mathbb{C}^{\text {static }}
$$

For instance, $\mathbb{C}^{\sin k}=\{$ communities 1,3 , and 4$\}$ in Figure 1 . Definition 2 [Sink-Aware (SA) Centrality)]. For a mobile relay $x \in \mathcal{R}$ with social profile $\left(\mathbb{C}_{x}, \boldsymbol{h}_{x}\right)$, its $S A$ centrality $H_{x}^{\text {sink }}$ is defined as

$$
H_{x}^{\text {sink }}=\sum_{\mathcal{C}_{x}^{i} \in \mathbb{C}^{\operatorname{sink} \cap} \cap \mathbb{C}_{x}} h_{x}^{i}
$$

where $h_{x}^{i}$ is the local centrality of $x$ in community $\mathcal{C}_{x}^{i}$.

It can be seen that a mobile relay with large global centrality (i.e. the sum of its all local centralities) may not have a large SA centrality. For instance, a policeman may meet many people and therefore has a large global centrality, yet he may rarely patrol the streets where the sinks are deployed. A numerical example is also shown in Figure1 (c), where the global centrality of relay $x$ is 9 which is larger than that of relay $y$ (i.e. 7). However, the SA centrality of $x$ is less than that of $y$ (i.e. $H_{x}^{\text {sink }}=6<H_{y}^{\text {sink }}=7$ ).

Let the maximal possible SA centrality over all mobile relays be $\mathrm{H}_{\text {sink }}^{\max }=\max _{x \in \mathcal{R}} H_{x}^{\text {sink }}$, it is obvious that $\mathrm{H}_{\text {sink }}^{\max } \leq$ $|\mathcal{R}|$. Although static nodes do not have centrality concept, we also assign a SA centrality value for each static node $x \in$ $\mathcal{S} \cup \mathcal{D}$

$$
H_{x}^{\text {sink }}= \begin{cases}|\mathcal{R}| & \text { if } x \in \mathcal{A} \in \mathbb{A}^{\text {sink }} \\ 0 & \text { otherwise }\end{cases}
$$

to support our OBSEA algorithm that seamlessly combines all static nodes and mobile relays in the whole WSN-HR. For instance, in Figure $1, H_{x}^{\text {sink }}=|\mathcal{R}|=18$, if a static node $x$ is in static clusters 2 and 3; otherwise $H_{x}^{\text {sink }}=|\mathcal{R}|=0$.

Compared with classic centrality definitions used in social networks and DTNs (e.g. degree and betweenness centralities [20], [46]), SA centrality provides the destination-awareness for mobile relays, and thus is more effective for WSN-HR.

\section{E. Economic Perspective: Incentive and Social Profits}

To incentivise mobile relays to forward the sensor data for the static WSN, we establish a virtual economic network for the WSN-HR. The static WSN $G^{w s n}\left(\mathcal{S} \cup \mathcal{D}, \mathcal{L}^{w s n}\right)$ can be considered as the employer who pays the mobile relays (the employees) in credits, which can be used for online shopping (e.g. to buy Android/iPhone Apps). Specifically, at each slot $t \geq 1$, the sensor data production and trading processes are described as follows:

- When a sensor or sink node $y \in \mathcal{S} \cup \mathcal{D}$ receives (buys) $f_{x, y}(t)$ amount of sensor data from a mobile relay $x \in \mathcal{R}$, $y$ pays $f_{x, y}(t) \lambda_{x}(t)$ amount of credits to $x$, where $\lambda_{x}(t)$ is selling price per unit data (decided by the seller $x$ ).

- When a mobile relay $x$ sells (transmits) data to another relay $y, x, y \in \mathcal{R}, x$ will receive a payment of $f_{x, y}(t)\left(\lambda_{x}(t)-\lambda_{y}(t)\right)$ amount credits from $y$.

- A mobile relay $y \in \mathcal{R}$ can receive data for free from any sensor node $x \in \mathcal{S}$.

- When a sensor node $x \in S$ collects environmental data at a sampling rate $r_{x}(t), I_{x}\left(r_{x}(t)\right)$ amount of revenue will be provided by the WSN, where $I_{x}\left(r_{x}(t)\right)$ can be any differentiable, non-decreasing, non-negative, and concave utility function of $r_{x}(t)$. 
The sub-network consists of mobile relays and can be viewed as a free information market, in which every relay trades sensor data with other relays to reap benefits from price differences; similar to the real-world business. The instantaneous profit of a mobile relay $x \in \mathcal{R}$ is defined as

$$
\gamma_{x}^{\text {relay }}(t)=\sum_{y \in \mathcal{N}_{x}(t)} \lambda_{x}(t) f_{x, y}(t)-\sum_{y \in \mathcal{N}_{x}(t) \cap \mathcal{R}} \lambda_{y}(t) f_{y, x}(t)
$$

where the first term in the right hand side of (5) represents the total revenue of $x$ by selling data to others, and the last term represents the total expenditure of $x$, i.e. the credits paid for data purchase from other relays in $\mathcal{R} \cap N_{x}(t)$. Similarly, we define the instantaneous profit of the static WSN as

$$
\Gamma^{w s n}(t)=\sum_{x \in \mathcal{S}} I_{x}\left(r_{x}(t)\right)-\sum_{x \in \mathcal{S} \cup \mathcal{D}} \sum_{y \in \mathcal{N}_{x}(t) \cap \mathcal{R}} \lambda_{y}(t) f_{y, x}(t)
$$

where $\sum_{x \in \mathcal{S}} I_{x}\left(r_{x}(t)\right)$ represents the total instantaneous revenue of the WSN, and the last term of (6) represents the total expenditure of the WSN, i.e. the total credits paid by the WSN for data purchase from mobile relays.

From (5) and (6), it is easy to verify that the instantaneous global social profit of the whole WSN-HR is

$$
\begin{aligned}
\Gamma(t) & =\sum_{x \in \mathcal{R}} \gamma_{x}^{\text {relay }}(t)+\Gamma^{\text {wsn }}(t) \\
& =\sum_{x \in S} I_{x}\left(r_{x}(t)\right)
\end{aligned}
$$

This is because the sum of the internal payments of all nodes in $\mathcal{N}$ is equal to the sum of revenue earned from taking these payments. Therefore, the total social profit is the total external incomes of the WSN.

\section{F. Social Profits Maximization}

Due to the arbitrary stochastic process of channel state (which may be non-ergodic) $\boldsymbol{c}(t)$, an infinite-horizon timeaverage of social profits may not exist. Therefore, we consider a finite number of slots $t \in\left\{1,2, \ldots, t_{\text {end }}\right\}$. The objective is to seek an algorithm to solve the following finite-horizon optimization problem:

$$
\max \quad \bar{\Gamma}=\frac{1}{t_{\text {end }}} \sum_{t=1}^{t_{\text {end }}} \Gamma(t)
$$

subject to

$$
\begin{aligned}
& 0 \leq r_{x}(t) \leq \mathrm{r}^{\max } \quad x \in \mathcal{S}, 1 \leq t \leq t_{\text {end }} \\
& Q_{x}(t) \leq Q_{x}^{\max }, \forall x \in \mathcal{S} \cup R, 1 \leq t \leq t_{\text {end }} \\
& 0 \leq f_{x, y}(t) \leq c_{x, y}(t) \leq \mathrm{c}^{\max } x, y \in \mathcal{N}, 1 \leq t \leq t_{\text {end }} \\
& \quad \sum_{y \in \mathcal{N}-\{x\}} \bar{f}_{x, y} \geq 1_{\{x \in \mathcal{S}\}} \bar{r}_{x}+\sum_{y \in \mathcal{N}-\{x\}} \bar{f}_{y, x}, x \in \mathcal{S} \cup \mathcal{R}
\end{aligned}
$$

where $\bar{f}_{x, y}=\sum_{t=1}^{t_{e n d}} f_{x, y}(t) / t_{\text {end }}$; and the indicator function $1_{\{x \in \mathcal{S}\}}=1$ if $x \in \mathcal{S}, 1_{\{x \in \mathcal{S}\}}=0$ otherwise. The objective (8) is to maximize the time average social profits of all mobile relays and the WSN during the finite-horizon of size $t_{\text {end }}$. The constraint (10) represents that the sample rate $r_{x}(t)$ is bounded by a constant value $\mathrm{r}^{\max }<\infty$, this is realistic for typical sensor nodes. The constraint (11) states the queue backlog $Q_{x}(t)$ of a sensor node or a mobile relay $x$ should be less than its buffer size $Q_{x}^{\max }$. The constraint (12) represents that the actual amount of data forwarded over each link should be not more than the capacity of this link. Constraint (13) states the flow conservation law, i.e. node $x$ 's average total incoming data rate must be not more than its average total outgoing data rate.

\section{OPPORTUNISTIC BACKPRESSURE WITH SOCIAL/ECONOMIC AWARENESS}

In this section, we introduce a simple distributed scheme to establish SA centrality and the OBSEA algorithm.

\section{A. SA Centrality Update}

The following simple GPS-free scheme can establish SA centrality for each mobile relay in a fully distributed way ${ }^{4}$.

Step 1. Every mobile relay $x \in \mathcal{R}$ initializes its SA centrality as $H_{x}^{\text {sink }}=0$.

Step 2. Each mobile relay $x \in \mathcal{R}$ establishes the social profile $\left(\mathbb{C}_{x}, \boldsymbol{h}_{x}\right)$ by using its online social neighbor table $\mathcal{N}_{x}^{\text {social }}$ and a distributed community detection algorithm [43].

Step 3. Each static node $x \in \mathcal{S} \cup \mathcal{D}$ maintains a set $\mathcal{F}_{x}=$ $\left\{y \mid \in \mathcal{R}, \overline{I C T}_{x, y}<\mathrm{ICT}{ }^{\max }\right\}$, i.e. the set of mobile relays that visit $x$ frequently. Then $x$ can establish $\mathbb{C}_{x}^{\text {static }}$ as

$$
\mathbb{C}_{x}^{\text {static }}=\bigcup_{y \in \mathcal{F}_{x},}\{\mathcal{C}\}
$$

Step 4. If a node $x \in \mathcal{S} \cup \mathcal{D}$ in a static cluster $\mathcal{A}$ that also contains one or multiple sinks, then $x$ broadcasts $\mathbb{C}_{x}^{\text {static }}$ to all other nodes in $\mathcal{A}$. As a result, every static node in $\mathcal{A}$ can know the set $\mathbb{C}_{\mathcal{A}}^{\text {static }}=\bigcup_{y \in \mathcal{A}} \mathbb{C}_{y}^{\text {static }}$, i.e. the set of communities whose geographic areas static cluster that $\mathcal{A}$ is located in. For instance, in Figure $1, \mathbb{C}_{\text {static }}^{\text {static }}$ cluster $2=\{$ communities 1 and 2\}.

Step 5. When a mobile relay $x \in \mathcal{R}$ visits a static node $y \in \mathcal{S} \cup \mathcal{D}$ in a static cluster $\mathcal{A}$ that contains a sink. $x$ checks whether $y$ meets any node in $\mathcal{A}$. If not, $x$ requires $\mathbb{C}_{\mathcal{A}}^{\text {static }}$ from $y$, and then updates its SA centrality as

$$
H_{x}^{\text {sink }}=H_{x}^{\text {sink }}+\sum_{\mathcal{C}_{x}^{i} \in \mathbb{C}_{\mathcal{A}}^{\text {static } \cap \mathbb{C}_{x}}} h_{x}^{i}
$$

\section{B. OBSEA Algorithm}

At each slot $t \geq 1$, each node $x \in \mathcal{N}$ first observes its current neighbors that it is in contact with $\mathcal{N}_{x}(t)$, the queue backlogs of itself and its contact neighbors, and channel capacities of all its outgoing links, $c_{x, y}(y), y \in \mathcal{N}_{x}(t)$. Then each node runs the OBSEA algorithm as follows:

Pricing. The selling price set by every node $x \in \mathcal{N}$ in slot $t$ is:

$$
\lambda_{x}(t)=\left(Q_{x}(t)+\alpha\left(\mathrm{H}_{\mathrm{sink}}^{\max }-H_{x}^{\text {sink }}\right)\right) / \lambda_{\text {scale }}
$$

${ }^{4}$ Due to the time-varying nature of human mobility patterns [6], [11], [44], each node operates steps 1-5 during every short-term period (e.g. six hours) to obtain the transient SA centrality rather than the long-term cumulative one. 
where $\lambda_{\text {scale }}>0$ is the price-scaling parameter that does not impact the global social profits, but controls the profit ratio between all mobile relays (all employees) and the static WSN (the employer), i.e. $\sum_{x \in \mathcal{R}} \gamma_{x}^{\text {relay }}(t) / \Gamma^{W S N}(t)$ ); and $\alpha \geq 0$ is the weighting parameter for SA centrality awareness in the routing component of OBSEA. When $\alpha=0$, the routing of OBSEA is the pure queue-backlog aware (backpressure) algorithm; as $\alpha \rightarrow \infty$, the the routing of OBSEA tends to be based on SA centrality only. It worth noting that the selling price $\lambda_{x}(t)$ is always non-negative, due to the non-negativity of $\alpha, Q_{x}(t), \lambda_{\text {scale }}$, and $\mathrm{H}_{\text {sink }}^{\max }-H_{x}^{\text {sink }}$.

Rate Control. Each sensor node $x \in \mathcal{S}$ sets its data sampling rate $r_{x}(t)$ to maximize the following simple problem.

$\max I_{x}\left(r_{x}(t)\right)-r_{x}(t) Q_{x}(t) / V$

subject to

$$
0 \leq r_{x}(t) \leq \mathrm{r}^{\max }
$$

where $V>0$ is the predefined control parameter for the tradeoff between queue backlogs and social profits. Since $I_{x}\left(r_{x}(t)\right)$ is concave, problem (15) adopts an unique maximizer as

$$
\widetilde{r}_{x}(t)=\min \left[\max \left[I_{x}^{\prime-1}\left(Q_{x}(t) / V\right), 0\right], \mathrm{r}^{\max }\right]
$$

where $I_{x}^{\prime-1}()$ represents the inverse function of the utility function $I_{x}()$ 's first derivative.

Routing. Recall that $Q_{x}^{\max }$ is the data buffer size of node $x$. Each node $x \in \mathcal{S} \cup \mathcal{R}$ computes the price differential between itself and each of its instantaneous contact neighbors $y \in \mathcal{N}_{x}(t)$, by using (14). Then $x$ computes the weight of instantaneous link as

$w_{x, y}(t)= \begin{cases}\left(\lambda_{x}(t)-\lambda_{y}(t)\right) \lambda_{\text {scale }} & \text { if } Q_{y}(t)<Q_{y}^{\max }-\eta_{y}(t) \\ 0 & \text { otherwise }\end{cases}$

where $\eta_{y}(t)=\sum_{z \in \mathcal{N}_{y}(t)} c_{z, y}(t)+1_{\{y \in \mathcal{S}\}} \mathrm{r}^{\max }$ is the largest possible amount of data that can be injected into node $y$ at slot $t$. Then node $x$ transmits $f_{x, y}(t)$ amount of data packets to $y$ :

$$
f_{x, y}(t)= \begin{cases}c_{x, y}(t) & \text { if } w_{x, y}(t)>0 \\ 0 & \text { otherwise }\end{cases}
$$

It is clear that sensor-data packets are dynamically forwarded hop-by-hop rather than through maintained end-to-end paths.

Incentive and Credit Transfer. Based on the actual produced and transmitted sensor data decided by above rate control and routing respectively, each node in $\mathcal{N}$ transfers the credits using the mechanism described in Subsection II-E.

All the static nodes in the WSN are enforced to obey the rate control and routing rule without any incentive. Now we analyze why mobile relays are willing to follow above routing rule. Consider $w_{x, y}(t)>0$, there are two cases:

Case 1. Both $x$ and $y$ are mobile relays. $x$ can achieve $\lambda_{x}(t) c_{x, y}(t)$ credits by selling sensor data to $y$. For node $y$, although it pays credits for buying sensor packets, its next-slot selling price $\lambda_{y}(t+1)$ will be increased due to the incremental of its queue backlog (see (14)). Therefore, $y$ can obtain more credits by selling sensor-data packets to others in the future.
Based on the rationally-selfish assumption of mobile relays, both nodes are willing to trade the sensor data at this contact.

Case 2. One is a static node and the other is a relay. If $x$ is a sensor node, then relay $y$ can get free data from $x$, which can be sold to others in the future; if $x$ is a relay, it can get benefit by selling the data.

Queue Update. Queue backlog of each node $\in \mathcal{N}$ are updated according to (1).

Since every node $x \in \mathcal{N}$ requires only the information of its instantaneous neighbors in $\mathcal{N}_{x}(t)$, the OBSEA algorithm is fully distributed. In addition, OBSEA is based on only current knowledge of the network at slot $t$ and does not require any prediction capacity for future knowledge after slot $t$.

\section{PERFORMANCE ANALYSis}

\section{A. Control Overhead}

The control overhead of the OBSEA algorithm is discussed as follows:

- Communication Overhead. Since OBSEA is fully distributed, each node only transmits at most one beacon to communicate its local queue backlog and SA centrality at each slot.

- Computational Overhead. Since a node $x \in \mathcal{N}$ can contact with at most $|\mathcal{N}|-1$ nodes at each slot, both the SA centrality update and the operations of OBSEA require at most $O(|\mathcal{N}|)$ simple arithmetic calculations only. It worth noting this is a loose bound, since a node can normally contact with a small number of nodes at each slot due to the sparse density of the network.

- Storage Overhead. Each node needs to maintain its SA centrality and its social profile (at most $2|\mathcal{N}|-1$ ) values. Therefore, the per node storage overhead is $\mathrm{O}(|\mathcal{N}|)$.

\section{B. Bounded Queues}

Memory is a key resource for both sensor nodes and mobile relays. Theorem 1 below shows that all data queue backlogs are deterministically bounded.

Theorem 1. Suppose the initial queue backlogs $Q_{x}(1)=$ $0, \forall x \in \mathcal{S} \cup \mathcal{R}$, then $Q_{x}(t)$ is always less than its buffer size $Q_{x}^{\max }, \forall t \geq 1$, if:

$$
Q_{x}^{\max } \geq \begin{cases}V I_{x}^{\prime}(0)+\eta^{\max } & x \in \mathcal{S} \\ \eta^{\max } & x \in \mathcal{R}\end{cases}
$$

where $\eta^{\max }=\max _{x \in \mathcal{S} \cup \mathcal{R}, t \geq 1} \eta_{x}(t) \leq|\mathcal{N}| \mathrm{c}^{\max }$. The proof of Theorem 1 can be found in Appendix. In practice, $\eta^{\max }, I_{x}^{\prime}(0)$, and $Q_{x}^{\max }, x \in \mathcal{S} \cup \mathcal{R}^{5}$ are normally fixed and can be determined in advance. Therefore, the parameter $V$ can be set to guarantee the inequality (18). For instance, if the utility function of a sensor node $x$ is chosen as $I_{x}\left(r_{x}(t)\right)=\ln \left(r_{x}(t)+1\right)$, then $I_{x}^{\prime}(0)=1$ and $V$ should be not larger than $Q_{x}^{\max }-\eta^{\max }$.

\footnotetext{
${ }^{5}$ Although we ignore wireless interference and assume full-duplex wireless radio in this paper for simplicity, most current wireless transceivers are halfduplex. Therefore, each node $x \in \mathcal{S} \cup R$ can simply set $\eta^{\max }$ as the data rate (in packets per slot) of its transceiver.
} 


\section{Social Profits performance}

To derive the performance bounds of our OBSEA scheme, we divide the duration $1 \leq t \leq t_{\text {end }}$ into $K$ frames with size of $T$ slots, i.e. $K T=t_{\text {end }}$. We assume that there exists an ideal algorithm that knows the full network information (i.e. the mobility trace and channel capacity) for the future $T$ slots. Based on future knowledge, the ideal algorithm solves the following optimization problem at the beginning of each frame $k \leq K$ :

$$
\max \quad \frac{1}{T} \sum_{t=k T}^{k T-T+1} \Gamma(t)
$$

\section{subject to}

$$
\begin{gathered}
0 \leq r_{x}(t) \leq \mathrm{r}^{\max } \forall x \in \mathcal{S}, \forall t \\
Q_{x}(t) \leq Q_{x}^{\max }, \forall x \in \mathcal{S} \cup R, \forall t \\
0 \leq f_{x, y}(t) \leq c_{x, y}(t), \forall x \in \mathcal{N}, y \in \mathcal{N}_{x}(t), \forall t \\
\frac{1}{T} \sum_{t=k T-T+1}^{k T}\left(\sum_{y \in \mathcal{N}-\{x\}} f_{x, y}(t)-1_{\{x \in \mathcal{S}\}} r_{x}(t)\right. \\
\left.-\sum_{y \in \mathcal{N}-\{x\}} f_{y, x}(t)\right) \geq 0, \forall x \in \mathcal{S} \cup \mathcal{R}, \forall k \leq K
\end{gathered}
$$

The objective (19) demonstrates that the ideal algorithm optimizes the social profits over each frame $1 \leq k \leq K$.

Define $\Gamma_{k}^{*}(T)$ as the optimal social profits of problem (19) for the $k$ th $T$-slot frame. Let $r_{x}^{*}(t), x \in \mathcal{S}$ and $f_{x, y}^{*}(t), x \in$ $\mathcal{N}, y \in \mathcal{N}_{x}(t)$ respectively be the rate control and routing decisions made by the ideal algorithm that achieve $\Gamma_{k}^{*}(T)$. Due to the requirement of complete future knowledge, it is impossible to design such an ideal algorithm to achieve $\Gamma_{k}^{*}(T)$ in practice. However, $\Gamma_{k}^{*}(T)$ can be used as a performance baseline.

Theorem 2. The average social profits of OBSEA algorithm, $\bar{\Gamma}$, satisfies:

$$
\bar{\Gamma}=\frac{1}{K T} \sum_{t=1}^{K T} \Gamma(t) \geq \frac{1}{K} \sum_{k=1}^{K} \Gamma_{k}^{*}(T)-\frac{M T+Z}{V}
$$

where

$$
\begin{aligned}
& M=\frac{1}{2}|\mathcal{N}|^{2}\left(\mathrm{c}^{\max }+\mathrm{r}^{\max }\right)^{2} \\
& Z=|\mathcal{N}|^{2} \mathrm{c}^{\max }\left(2 \alpha \mathrm{H}_{\text {sink }}^{\max }+\eta^{\max }\right)
\end{aligned}
$$

The proof of Theorem 2 can be found in the Appendix. Inequality (25) shows that the average social profits of our OBSEA algorithm will not be smaller than that of the ideal algorithm minus a term $(M T+Z) / V$ during a finite horizon with size $t_{\text {end }}$. In addition, the constraint (24) is more stringent than the constraint (13), since (24) requires flow conservation law over each $T$-slot frame, rather than over total $K$ frames. Therefore, $\frac{1}{K} \sum_{k=1}^{K} \Gamma_{k}^{*}(T)$ is not larger than the optimal solution of problem (8). Therefore, the global social profit achieved by our OBSEA algorithm is not necessary smaller than that of the ideal algorithm, $\frac{1}{K} \sum_{k=1}^{K} \Gamma_{k}^{*}(T)$.
As $M$ and $Z$ are constant, parameter $V$ can be set as large as desired to enforce $(M T+Z) / V$ to be arbitrarily small, but resulting a large risk of packet loss caused by data buffer overflow, according to Theorem 1 . In practice, $V$ can be chosen as gfg

$$
V=\min _{x \in \mathcal{S}}\left(Q_{x}^{\max }-\eta^{\max }\right) / I_{x}^{\prime}(0)
$$

to maximize the worst-case global social profit while guaranteeing no packet loss caused by buffer overflow.

\section{Simulation}

We evaluate the practical performance of OBSEA algorithm in Castalia [15], a realistic WSN simulator. Given most current commercial short-range commercial radios (e.g. WiFi Direct and ZigBee) are based on CSMA, we implemented OBSEA on the top of CSMA link layers, by using the distributed scheduling scheme in [47].

Several real human mobility traces exist (e.g. [41]), however, public GPS data for WSN is non-existent. To integrate mobile relays and static WSNs into a geographic area, therefore, we constructed a WSN-HR that consists of a random deployed static WSN and multiple mobile relays that follow the Heterogeneous Human Walk (HHW) mobility model [11]. HHW is a realistic human mobility model based on social network theory, which exhibits various features of real human mobility and social networks.

The size of the geographic area was set as $1.7 \times 1.7 \mathrm{~km}^{2}$ which is approximately the same size of the City of London. The total number of nodes was set as 100 , which consists of 17 sensor nodes, 3 sinks, and 80 mobile relays. We set the duration of a slot to 1 second and ran the simulation for $10^{6}$ slots (the equivalent of about 11 days). We considered the 4-clique community structure for the HHW mobility model and set the parameters as $\mathrm{PR}^{\mathrm{Csize}}=1.2, \mathrm{PR}^{\text {Osize }}=2$, $\mathrm{PR}^{\text {Csize }}=1.2, \mathrm{PR}^{\mathrm{MN}}=2$, and $\mathrm{PR}^{\text {Local }}=2$, according to the observations of real social networks and human mobility traces. In addition, the speed of each mobile relay was randomly distributed between 0 and $10 \mathrm{~m} / \mathrm{s}$ (between walking and urban vehicular speeds.).

The transmission ranges of all nodes were set as $\mathbf{5 0}$ meters, and the data forwarding rate (capacity) of each instantaneous contact link is randomly selected between 1 and 20 packets per second. We set $\mathrm{r}^{\max }=10, \eta^{\max }=50$, and $\mathrm{H}_{\mathrm{sink}}^{\max }=80$. For each sensor node $x \in \mathcal{S}$, we set its buffer size $Q_{x}^{\max }=150$, and utility function $I\left(r_{x}(t)\right)=20 \ln \left(1+r_{x}(t)\right)$. The profitbacklog tradeoff parameter $V$ was set as 5 according to (28).

Figure 2 shows the average global social profit, network throughput, end-to-end delay, and queue backlogs with different weighting parameter $\alpha$ in (14), where $\mathrm{Q}_{\text {relay }}^{\max }$ represents the data buffer size of each mobile relay, i.e. $Q_{x}^{\max }=$ $\mathrm{Q}_{\text {relay }}^{\max }, \forall x \in \mathcal{R}$. Here, $\mathrm{Q}_{\text {relay }}^{\max }$ can be understood as either the physical memory size of mobile phones, or the memory space that the phone-user is willing to provide to the sensor data. Maximal social profits and network throughput are achieved when OBSEA sets $\alpha=10,100$, and 1000 for $\mathrm{Q}_{\text {relay }}^{\max }=150$, 300 , and 600 respectively. In addition, the OBSEA algorithm 


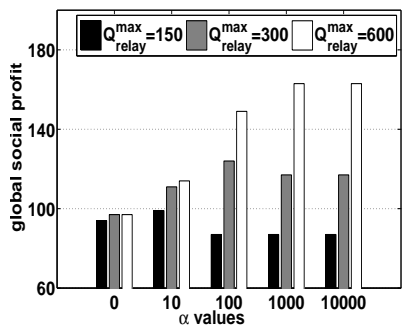

(a) global social profit

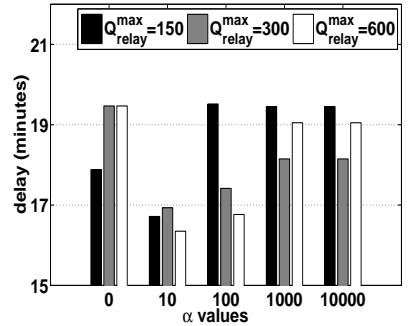

(c) per packet end-to-end delay

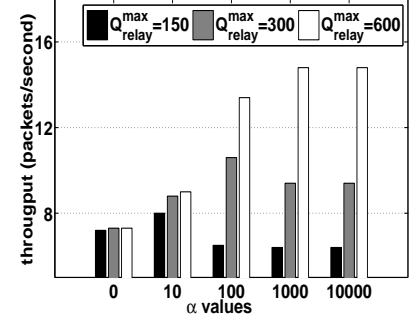

(b) network throughput

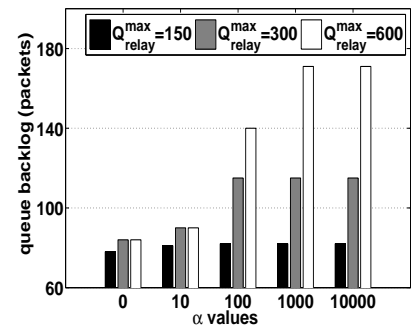

(d) average queue backlog
Fig. 2. Long-term average simulation results for different $\alpha$ and $\mathrm{Q}_{\text {relay }}^{\max }$. (a) The object of this paper $\bar{\Gamma}$, which can be transferred to various units such as pounds or dollars per day. (b) The sum of the packet receiving rates of all sinks. (c) The average sensor-to-sink delivery delay of all generated sensordata packets during the simulation. (d) Average queue backlog of all sensor nodes and mobile relays during the simulation.

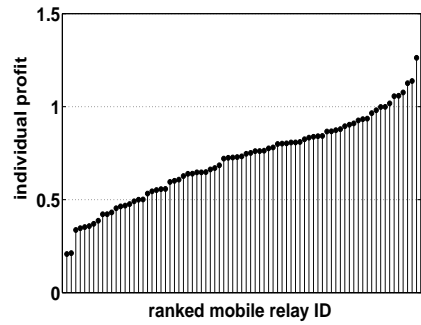

(a) average profit of each relay

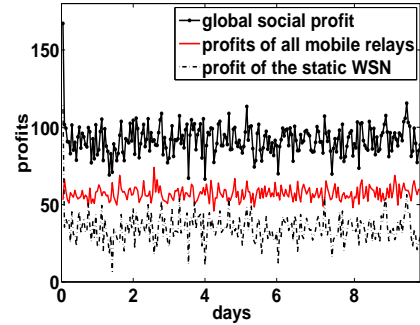

(b) dynamic social profits
Fig. 3. Distribution of average individual profit and dynamic global social profit for 10 days, with $\mathrm{Q}_{\text {relay }}^{\max }=600, \alpha=100$, and $\lambda_{\text {scale }}=10^{4}$.

shows minimal end-to-end delay when $\alpha=10$ for all simulations. The results above demonstrate that neither the pure backlog-aware scheme $(\alpha=0)$ nor the (approximately) pure social-aware scheme $(\alpha=10000)$ can achieve the optimal performance in all network conditions. However, OBSEA can adapt to different network settings, by simply adjusting the weighting parameter $\alpha$.

Figure 3 (a) shows that the profit of every mobile relay is positive, which means that every phone user achieves benefits through relaying sensor data for the static WSNs. The phone users just need to allocate some memory space $Q_{\text {relay }}^{\max }$ and incur minimal power consumption for short-range data transmissions to achieve such benefits. Figure 3 (b) shows that the "win-win situation" (non-negative benefits) is achieved for both static WSNs and mobile relays during every hour of the simulation time. We have also run the other simulations with different parameter settings, all of them show similar features to the results in Figures 2 and 3.

\section{CONCLUSION}

This paper combines network science principles and Lyapunov network optimization theory to develop a data muling scheme for sparse sensor networks with mobile phones. Without making any assumptions regarding the topology, mobility, and channel conditions of the network, we formalize a finite-horizon optimization problem for joint rate control, opportunistic routing, and resource pricing, which maximizes the global social profit of the network. By exploiting the social and economic behaviors of mobile phone users, a lightweight algorithm, OBSEA, is proposed that solves the formalized problem in a fully distributed manner. We prove that all queue backlogs are deterministically bounded, and the social profit achieved is close to, or better than, an ideal algorithm with perfect future knowledge. Simulation results show that OBSEA is adaptive to different network scenarios and outperforms pure backpressure and pure social-aware schemes.

Interesting future directions includes privacy issues, applying queueing-awareness for epidemic-style routing schemes, as well as considering other traffic communication patterns in WSN-HRs such as multicasting, sensor information queries, and publish/subscribe patterns.

\section{APPENDIX}

We present Lemma 1 to support the proof of Theorem 1 . Lemma 1. Considering a sensor node $x \in \mathcal{S}$ at a slot $t \geq 1$, if $Q_{x}(t) \geq V I_{x}^{\prime}(0)$, then the rate controller of OBSEA algorithm sets $r_{x}(t)=0$.

Proof. Since $I_{x}(r(t))$ is concave, its first derivative $I_{x}^{\prime}(r(t))$ is a monotonically decreasing function of $r(t)$. Therefore, we have

$$
I_{x}(r(t)) \leq I_{x}(0)+I_{x}^{\prime}(0) r_{x}(t), \forall 0 \leq r_{x}(t) \leq \mathrm{r}^{\max }
$$

Considering (29) and the objective of the rate controller (15), we have for any $0 \leq r_{x}(t) \leq \mathrm{r}^{\max }$

$$
\begin{aligned}
& I_{x}(r(t))-Q_{x}(t) r_{x}(t) / V \\
& \leq I_{x}(0)-r_{x}(t)\left(Q_{x}(t) / V-I_{x}^{\prime}(0)\right) \\
& \leq I_{x}(0)
\end{aligned}
$$

Inequality (30) holds only when $r_{x}(t)=0$, as $Q_{x}(t) / V-$ $I_{x}^{\prime}(0)>0$ (the condition of Lemma 1). Then, the rate controller must set $r_{x}(t)=0$ to maximize (15).

Proof of Theorem 1. We prove Theorem 1 by using mathematical induction. From the supposition of Theorem 1, we have $Q_{x}(1) \leq \mathrm{Q}^{\max }$ hold at slot 1 . Suppose $Q_{x}(t) \leq Q_{x}^{\max }$ for a slot $t \geq 2$, then there are two cases :

Case 1. $Q_{x}(t) \leq Q_{x}^{\max }-\eta_{x}(t), x \in \mathcal{S} \cup \mathcal{R}$. Since $\eta_{x}(t)$ is the maximum possible amount of data that can be injected in to node $x$ at slot $t$, it is clear that $Q_{x}(t+1)<Q_{x}^{\max }$ at slot $t+1$, according to (1).

Case 2. $Q_{x}^{\max }-\eta_{x}(t)<Q_{x}(t) \leq Q_{x}^{\max }, x \in \mathcal{S} \cup \mathcal{R}$. In this case, the link weight $w_{z, x}(t)$ will be assigned as 0 , for all $x$ 's instantaneous neighbors $z \in \mathcal{N}_{x}(t)$, according to (17). Hence, no data will be transmitted to $x$, according to (17). Therefore, if $x$ is a mobile relay, then $Q_{x}(t+1) \leq$ $Q_{x}(t) \leq Q_{x}^{\max }, x \in \mathcal{R}$. Further, if $x$ is a sensor node, since 
$Q_{x}(t)>Q_{x}^{\max }-\eta_{x}(t) \geq V I_{x}^{\prime}(0)+\eta^{\max }-\eta_{x}(t)>V I_{x}^{\prime}(0)$, we have $r_{x}(t)=0$, according to Lemma 1 the the condition of Theorem 1. Therefore, we can see that $Q_{x}(t+1) \leq Q_{x}(t) \leq$ $Q_{x}^{\max }, x \in \mathcal{S}$. In summary, $Q_{x}(t+1) \leq Q_{x}^{\max }, \forall x \in \mathcal{S} \cup \mathcal{R}$.

Because $Q_{x}(t+1) \leq Q_{x}^{\max }, \forall x \in \mathcal{S} \cup \mathcal{R}$ in both cases, we can conclude that $Q_{x}(t) \leq Q_{x}^{\max }$ for all $t \geq 1$.

Proof of Theorem 2. Let $\boldsymbol{Q}(t)$ be the vector of all queues maintained at all nodes in $\mathcal{N}$. To simplify the proof, we assume the initial queue backlogs $\boldsymbol{Q}(0)=0$. Define the Lyapunov function $L(\boldsymbol{Q}(t))$ as

$$
L(t)=\frac{1}{2} \sum_{x \in \mathcal{N}} Q_{x}^{2}(t)
$$

Denote $\Delta H_{x, y}^{\text {sink }}=H_{x}^{\text {sink }}-H_{y}^{\text {sink }}$ and $\Delta Q_{x, y}(t)=$ $Q_{x}(t)-Q_{y}(t)$. We first consider 1-slot drift-plus-penalty for each slot $1 \leq t \leq t_{\text {end }}$ :

$$
\begin{aligned}
& \triangle_{1} L(t)-V \Gamma(t) \\
& =L(t+1)-L(t)-V \Gamma(t) \\
& =\frac{1}{2} \sum_{x \in \mathcal{N}} Q_{x}^{2}(t+1)-\frac{1}{2} \sum_{x \in \mathcal{N}} Q_{x}^{2}(t)-V \Gamma(t) \\
& \leq_{a} M+\sum_{x \in \mathcal{N}} Q_{x}(t)\left(r_{x}(t) 1_{x \in \mathcal{S}}+\sum_{y \in \mathcal{N}_{x}(t)} f_{y, x}(t)\right. \\
& \left.-\sum_{y \in \mathcal{N}_{x}(t)} f_{x, y}(t)\right)-V \Gamma(t) \\
& =M+\sum_{x \in \mathcal{S}} r_{x}(t) Q_{s}(t)-V I_{x}(t) \\
& -\alpha \sum_{x \in \mathcal{N}_{, y \in \mathcal{N}_{x}(t)}} f_{x, y}(t) \Delta H_{x, y}^{\text {sink }} \\
& -\sum_{x \in \mathcal{N}, y \in \mathcal{N}_{x}(t)} 1_{\left\{Q_{y}(t)>Q_{y}^{\max }\right\}} f_{x, y}(t)\left(\triangle Q_{x, y}(t)\right.
\end{aligned}
$$

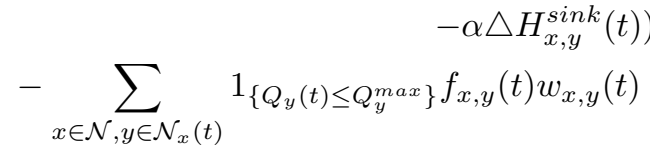

$$
\begin{aligned}
& \leq_{b} M+Z-V \sum_{x \in \mathcal{S}}\left(I_{x}(t)-r_{x}(t) Q_{s}(t) / V\right) \\
& -\sum_{x \in \mathcal{N}, y \in \mathcal{N}_{x}(t)} 1_{\left\{Q_{y}(t) \leq Q_{y}^{\max }\right\}} f_{x, y}(t) w_{x, y}(t)(32)
\end{aligned}
$$

where inequality $\leq_{a}$ followed by the fact that $M \geq$ $\sum_{x \in \mathcal{N}}\left(Q_{x}(t+1)-Q_{x}(t)\right)^{2}, \forall t$; and inequality $\leq_{b}$ is because of the following fact

$$
\begin{aligned}
Z= & |\mathcal{N}|^{2} c^{\max }\left(2 \alpha \mathrm{H}_{\text {sink }}^{\max }+\eta^{\max }\right) \\
= & \alpha|\mathcal{N}|^{2}\left(\mathrm{c}^{\max } \mathrm{H}_{\text {sink }}^{\max }\right)+|\mathcal{N}|^{2} \mathrm{c}^{\max }\left(\eta^{\max }+\alpha \mathrm{H}_{\text {sink }}^{\max }\right) \\
\geq & -\alpha \sum_{x \in \mathcal{N}, y \in \mathcal{N}_{x}(t)} f_{x, y}(t) \Delta H_{x, y}^{\text {sink }} \\
& \quad-\sum_{x \in \mathcal{N}, y \in \mathcal{N}_{x}(t)} 1_{\left\{Q_{y}(t)>Q_{y}^{\max }\right\}} f_{x, y}(t)\left(\triangle Q_{x, y}(t)\right. \\
& \left.\quad-\alpha \triangle H_{x, y}^{\text {sink }}(t)\right)
\end{aligned}
$$

It is easy to see that our OBSEA algorithm greedily minimizes the right-hand side of inequality (32) at every slot $t$, i.e. the rate controller minimizes the third term of the right-hand side of inequality (32), and the routing component minimizes the last term. Now we define the $T$-slot sample-path drift-plus-penalty for a frame $1 \leq k \leq K$

$$
\begin{aligned}
& \triangle_{T} L(k T)-V \sum_{t=k T-T+1}^{k T} \Gamma(t) \\
& =L(k T)-L(k T-T+1)-V \sum_{t=k T-T+1}^{k T} \Gamma(t) \\
& =\sum_{t=k T-T+1}^{k T}\left(\triangle_{1} L(t)-V \Gamma(t)\right) \\
& \leq{ }_{a} M T+Z T+\sqrt{2 M} \frac{T(T-1)}{2} \\
& +\sum_{x \in \mathcal{N}} \sum_{t=k T-T+1}^{k T} 1_{\left\{Q_{x}(t) \leq Q_{x}^{\max \}} Q_{x}(t)\left(r_{x}(t) 1_{x \in \mathcal{S}}\right.\right.} \\
& \left.+\sum_{y \in \mathcal{N}_{x}(t)} f_{x, y}(t)-\sum_{y \in \mathcal{N}_{x}(t)} f_{y, x}(t)\right)-V \sum_{t=k T-T+1}^{k T} \Gamma(t) \\
& \leq{ }_{b} M T^{2}+Z T \\
& \left.+\sum_{x \in \mathcal{N}} \sum_{t=k T-T+1}^{k T} 1_{\left\{Q_{x}(t) \leq Q_{x}^{\max \}} Q_{x}(t)\left(r_{x}^{*}(t) 1_{x \in \mathcal{S}}\right.\right.} f_{y \in \mathcal{N}-\{x\}} f_{x, y}^{*}(t)-\sum_{y \in \mathcal{N}-\{x\}} f_{y, x}^{*}(t)\right)-V T \Gamma^{*}(t) \\
& +\leq_{c} M T^{2}+Z T-V T \Gamma_{k}^{*}(T)
\end{aligned}
$$

where the inequality $\leq_{a}$ is based on inequality (32), the sum of $\triangle_{1} L(t)-V \Gamma(t)$ over $T$ slots, and the fact that the each queue backlog does not change by more than $(t-(k T-T+1))\left(\mathrm{r}^{\max }+\mathrm{c}^{\max }\right)$ for any slot $k T-T+1 \leq$ $t \leq k T$; the inequality $\leq_{b}$ is because $M \geq \sqrt{M}(M \geq 1)$, and our OBSEA algorithm minimizes the right-hand side of $\leq_{a}$ over all possible rate control and routing decisions, including the decisions of the ideal algorithm, $r_{x}^{*}(t), x \in \mathcal{S}$ and $f_{x, y}^{*}(t), x \in \mathcal{N}, y \in \mathcal{N}_{x}(t)$ that achieves $\Gamma_{k}^{*}(T)$; the inequality $\leq_{c}$ follows from the fact that the decisions $r_{x}^{*}(t)$ and $f_{x, y}^{*}(t)$ satisfy constraints (22) and (24).

The result of Theorem 2, inequality (25) can be obtained by taking a telescopic sum of the inequality (33) over $k \in$ $\{1, \ldots, K\}$, dividing both sides by $V K T$, considering the fact $L(1)=0$ and $L(K T+T) \geq 0$, and rearranging the terms.

\section{REFERENCES}

[1] A. Barabási and R. Albert, "Emergence of scaling in random networks," Science, vol. 286, no. 5439, pp. 509-512, 1999.

[2] D. Watts and S. Strogatz, "Collective dynamics of small-world networks," Nature, vol. 393, no. 6684, pp. 440-442, 1998.

[3] G. Palla, I. Derényi, I. Farkas, and T. Vicsek, "Uncovering the overlapping community structure of complex networks in nature and society," Nature, vol. 435, no. 7043, pp. 814-818, 2005.

[4] S. Fortunato, "Community detection in graphs," Physics Reports, vol. 486, no. 3-5, pp. 75-174, 2010.

[5] Q. Li, S. Zhu, and G. Cao, "Routing in socially selfish delay tolerant networks," in Proc. IEEE Infocom, 2010, pp. 1-9.

[6] W. Gao, G. Cao, T. La Porta, and J. Han, "On exploiting transient social contact patterns for data forwarding in delay tolerant networks," IEEE Trans. Mobi. Comput., no. 99, pp. 1-1, 2011.

[7] M. Conti, S. Giordano, M. May, and A. Passarella, "From opportunistic networks to opportunistic computing," IEEE Commun. Mag., vol. 48, no. 9, pp. 126-139, 2010. 
[8] U. Park and J. Heidemann, "Data muling with mobile phones for sensornets," in Porc. ACM Sensys, 2011, pp. 162-175.

[9] M. Conti and M. Kumar, "Opportunities in opportunistic computing," Computer, vol. 43, no. 1, pp. 42-50, 2010

[10] M. O'Grady and G. O'Hare, "How smart is your city?" Science, vol. 335, no. 6076, pp. 1581-1582, 2012.

[11] S. Yang, X. Yang, C. Zhang, and E. Spyrou, "Using social network theory for modeling human mobility," IEEE Network, vol. 24, no. 5, pp. 6-13, 2010.

[12] M. Chiang, S. Low, A. Calderbank, and J. Doyle, "Layering as optimization decomposition: A mathematical theory of network architectures," Proc. IEEE, vol. 95, no. 1, pp. 255-312, 2007.

[13] L. Georgiadis, M. Neely, M. Neely, and L. Tassiulas, Resource allocation and cross-layer control in wireless networks. Now Pub, 2006.

[14] Y. Yi and M. Chiang, "Stochastic network utility maximisation-a tribute to kelly's paper published in this journal a decade ago," European Transactions on Telecommunications, vol. 19, no. 4, pp. 421-442, 2008.

[15] http://castalia.npc.nicta.com.au/.

[16] J. Burrell, T. Brooke, and R. Beckwith, "Vineyard computing: Sensor networks in agricultural production," IEEE Pervasive Comput., vol. 3, no. 1, pp. 38-45, 2004.

[17] J. Huang, S. Amjad, and S. Mishra, "Cenwits: a sensor-based loosely coupled search and rescue system using witnesses," in Proc. ACM SenSys, 2005, pp. 180-191.

[18] M. Dunbabin, P. Corke, I. Vasilescu, and D. Rus, "Data muling over underwater wireless sensor networks using an autonomous underwater vehicle," in Proc. IEEE ICRA, 2006, pp. 2091-2098.

[19] O. Tekdas, V. Isler, J. Lim, and A. Terzis, "Using mobile robots to harvest data from sensor fields," IEEE Wireless Commun., vol. 16, no. 1 , pp. 22-28, 2009.

[20] P. Hui, J. Crowcroft, and E. Yoneki, "Bubble rap: social-based forwarding in delay tolerant networks," in Proc. ACM Mobihoc, 2008, pp. 241250 .

[21] W. Gao, Q. Li, B. Zhao, and G. Cao, "Social-aware multicast in disruption-tolerant networks," IEEE/ACM Trans. Netw., vol. 20, no. 5, pp. 1553-1566.

[22] K. Fall, "A delay-tolerant network architecture for challenged internets," in Proc. ACM Sigcomm, 2003, pp. 27-34.

[23] J. Ryu, V. Bhargava, N. Paine, and S. Shakkottai, "Back-pressure routing and rate control for icns," in Proc. ACM Mobicom, 2010, pp. 365-376.

[24] J. Ryu, L. Ying, and S. Shakkottai, "Back-pressure routing for intermittently connected networks," in Proc. IEEE Infocom, 2010, pp. 1-5.

[25] M. Neely and R. Urgaonkar, "Optimal backpressure routing for wireless networks with multi-receiver diversity," Ad Hoc Networks, vol. 7, no. 5, pp. 862-881, 2009

[26] L. Ying, S. Shakkottai, A. Reddy, and S. Liu, "On combining shortestpath and back-pressure routing over multihop wireless networks," IEEE/ACM Trans. Netw., vol. 19, no. 3, pp. 841-854, 2011.

[27] L. Le, E. Modiano, and N. Shroff, "Optimal control of wireless networks with finite buffers," in Proc. IEEE Infocom, 2010, pp. 1-9.

[28] M. Neely, "Universal scheduling for networks with arbitrary traffic, channels, and mobility," in Proc. IEEE CDC, 2010, pp. 1822-1829.

[29] M. Neely and L. Golubchik, "Utility optimization for dynamic peer-topeer networks with tit-for-tat constraints," in Proc. IEEE Infocom, 2011, pp. $1458-1466$.

[30] M. Neely, A. Tehrani, and A. Dimakis, "Efficient algorithms for renewable energy allocation to delay tolerant consumers," in Proc. IEEE SmartGridComm, 2010, pp. 549-554.

[31] M. Neely, "Optimal pricing in a free market wireless network," in Proc. IEEE Infocom, 2007, pp. 213-221.

[32] F. Kelly, A. Maulloo, and D. Tan, "Rate control for communication networks: shadow prices, proportional fairness and stability," Journ. of the Operational Res. society, vol. 49, no. 3, pp. 237-252, 1998.

[33] R. Ma, S. Lee, J. Lui, and D. Yau, "Incentive and service differentiation in p2p networks: a game theoretic approach," IEEE/ACM Trans. on Netw., vol. 14, no. 5, pp. 978-991, 2006.

[34] D. Niyato and E. Hossain, "Competitive pricing for spectrum sharing in cognitive radio networks: Dynamic game, inefficiency of nash equilibrium, and collusion," IEEE J. Sel. Areas Commun., vol. 26, no. 1, pp. 192-202, 2008.

[35] M. Rasti, A. Sharafat, and B. Seyfe, "Pareto-efficient and goal-driven power control in wireless networks: A game-theoretic approach with a novel pricing scheme," IEEE/ACM Trans. Netw., vol. 17, no. 2, pp. 556-569, 2009.

[36] R. Lu, X. Lin, H. Zhu, X. Shen, and B. Preiss, "Pi: A practical incentive protocol for delay tolerant networks," IEEE Trans. Wireless Commun., vol. 9, no. 4, pp. 1483-1493, 2010
[37] U. Shevade, H. Song, L. Qiu, and Y. Zhang, "Incentive-aware routing in dtns," in Proc. IEEE ICNP, 2008, pp. 238-247.

[38] A. Dvir and A. Vasilakos, "Backpressure-based routing protocol for dtns," in ACM SIGCOMM Computer Communication Review, vol. 40, no. 4, 2010, pp. 405-406.

[39] Q. Li, S. Zhu, and G. Cao, "Routing in socially selfish delay tolerant networks," in Proc. IEEE Infocom, 2010, pp. 1-9.

[40] U. Akyol, M. Andrews, P. Gupta, J. Hobby, I. Saniee, and A. Stolyar, "Joint scheduling and congestion control in mobile ad-hoc networks," in Proc. IEEE Infocom, 2008, pp. 619-627.

[41] A. Chaintreau, P. Hui, J. Crowcroft, C. Diot, R. Gass, and J. Scott, "Impact of human mobility on opportunistic forwarding algorithms," IEEE Trans. Mobi. Comput., vol. 6, no. 6, pp. 606-620, 2007.

[42] T. Karagiannis, J. Le Boudec, and M. Vojnovic, "Power law and exponential decay of intercontact times between mobile devices," IEEE Trans. Mobi.Comput., vol. 9, no. 10, pp. 1377-1390, 2010.

[43] P. Hui, E. Yoneki, S. Chan, and J. Crowcroft, "Distributed community detection in delay tolerant networks," in Proc. MobiArch, 2007.

[44] W. Hsu, T. Spyropoulos, K. Psounis, and A. Helmy, "Modeling timevariant user mobility in wireless mobile networks," in Proc. IEEE Infocom, 2007, pp. 758-766.

[45] M. Gonzalez, C. Hidalgo, and A. Barabási, "Understanding individual human mobility patterns," Nature, vol. 453, no. 7196, pp. 779-782, 2008.

[46] L. Freeman, "Centrality in social networks conceptual clarification," Social networks, vol. 1, no. 3, pp. 215-239, 1979

[47] U. Akyol, M. Andrews, P. Gupta, J. Hobby, I. Saniee, and A. Stolyar, "Joint scheduling and congestion control in mobile ad-hoc networks," in Proc. IEEE Infocom, 2008, pp. 619-627. 